\title{
CORONAVÍRUS: UMA REVISÃO HISTÓRICA E BIBLIOGRÁFICA
}

\author{
CORONAVIRUS: A HISTORICAL AND BIBLIOGRAPHIC REVIEW
}

\author{
Paloma Medeiros Gomes Cavalcanti ${ }^{1}$ \\ Ana Flávia Henriques Ribeiro Monteiro ${ }^{2}$ \\ Ana Paula Monteiro do Nascimento ${ }^{3}$ \\ Lucas Galvão Araújo ${ }^{4}$ \\ Mateus Louis Rodrigues Cavalcante ${ }^{5}$ \\ Raissa Cavalcanti Rodrigues ${ }^{6}$
}

RESUMO: OBJETIVO: Fazer uma retrospectiva histórica sobre os Coronavírus e compilar as informações de artigos até a data de realização deste trabalho a respeito do surto do novo Coronavírus, iniciado em dezembro de 2019. MÉTODO: Trata-se de uma revisão bibliográfica de caráter retrospectivo, realizado por meio da consulta a bases de dados do site da Organização Mundial de Saúde (OMS), PubMed, Google Acadêmico e LILACS. RESULTADOS: Embora a maioria das infecções por coronavírus humano sejam leves, as epidemias do coronavírus da síndrome respiratória aguda grave (SARS-CoV) e coronavírus da síndrome respiratória do Oriente Médio (MERS-CoV), causaram mais de 10.000 casos cumulativos nas últimas duas décadas, com taxas de mortalidade de $10 \%$ para SARS-CoV e $37 \%$ para MERS-Co. O 2019- nCoV vem se assemelhando com estes dois tipos mais agressivos do vírus, gerando um estado de alerta no mundo assim como ocorreu com essas doenças nos seus períodos de surto global. CONCLUSÃO: Diante do exposto, os vírus do gênero Coronavírus, desde o início do século vêm levantando estados de alerta de saúde para a população mundial e essa nova pandemia vem gerando medo e insegurança na população global, porém, com a experiência de epidemias e pandemias passadas, muitas medidas já foram tomadas, desde seu início, para minimizar seu impacto, mesmo que ainda desconhecido.

Palavras chave: Coronaviridae. Coronavirus. Infecções por Coronavirus.

\footnotetext{
${ }^{1}$ Estudante do Oitavo período de Medicina do Centro Universitário de João Pessoa - UNIPÊ.

${ }^{2}$ Estudante do Oitavo período de Medicina do Centro Universitário de João Pessoa - UNIPÊ.

${ }^{3}$ Estudante do Oitavo período de Medicina do Centro Universitário de João Pessoa - UNIPÊ.

${ }^{4}$ Estudante do Oitavo período de Medicina do Centro Universitário de João Pessoa - UNIPÊ.

${ }^{5}$ Estudante do Décimo primeiro Período de Medicina do Centro Universitário de João Pessoa UNIPÊ.

${ }^{6}$ Estudante do Quinto período de Medicina do Centro Universitário de João Pessoa - UNIPÊ.
} 
ABSTRACT: OBJECTIVE: To make a historical retrospective on the Coronavirus and compile as information from articles up to performance data of this work and respect the new Coronavirus, started in December 2019. METHOD: This is a retrospective bibliographic review, carried out through the consult databases on the World Health Organization (WHO) website, PubMed, Google Scholar and LILACS. RESULTS: Although most human coronavirus infections are mild, such as epidemics of severe acute respiratory syndrome (SARS-CoV) and Middle East respiratory syndrome (MERS-CoV) viruses, they cause more than 10,000 cumulative cases in past two decades, with mortality rates of $10 \%$ for SARS-CoV and $37 \%$ for MERS-Co. 2019-nCoV has been similar to these two more aggressive types of viruses, generating a state of alert in the world, just as occurred with these diseases in their global damage. CONCLUSION: Given the above, the viruses of the genus Coronavirus, since the beginning of the century, raising health alert states for a world population and this new pandemic has been generating fear and insecurity in the global population, however, with an experience of epidemics and pandemics In the past, many steps have been taken, from the beginning, to reduce the impact, even if it is still unknown.

Keywords: Coronaviridae. Coronavirus. Coronavirus infections. 


\section{INTRODUÇÃO}

Historicamente, com o surgimento de epidemias globais, surge junto com elas o sentimento de medo e insegurança na população, devido o grande impacto e repercussão gerado na sociedade como um todo. Podemos citar algumas pandemias que aterrorizaram a população mundial, como a "Praga de Justiniano" no século VI, a "Peste Negra" no século XIV, a "Gripe Espanhola" no século XX, a gripe aviária no início do século $\mathrm{XXI}$, a síndrome respiratória aguda grave (SARS-CoV) entre 2002 e 2004, a gripe suína, H1N1, em 2009, a síndrome respiratória do Oriente Médio (MERS-CoV) entre 2012 e 2015, a poliomielite em 2014, o Zika vírus e Ebola em 2016 e novamente em 2018, o Ebola. Atualmente, no início de 2020, estamos observando o impacto sociodemográfico causado pela rápida difusão internacional do novo coronavírus (2019 n-CoV), com centenas de morte, levando a OMS a declarar situação de emergência de saúde internacional (SENHORAS, 2020).

Os Coronavírus fazem parte de um gênero da família Coronaviridae, que inclui o gênero Coronavirus e Torovirus (PEIRIS et al., 2003). No início do século XXI, três grupos sorologicamente distintos de coronavírus eram conhecidos, identificados em animais e seres humanos, causando uma variedade de doenças graves. Esses vírus são divididos em três sorotipos: grupos 1, 2 e 3, sendo os do grupo 2 homólogos ao do vírus influenza C (MARRA, 2003). Além disso, os coronavírus humanos caracterizam-se por serem transmitidos predominantemente no inverno (HOEK et al., 2004).

Essa diversidade de coronavírus é facilitada pela infidelidade da RNA polimerase dependente de RNA, a alta frequência de recombinação de RNA e os genomas incomumente grandes para vírus de RNA. O que também facilita a daptação do organismo a novos hospedeiros e nichos ecológicos, às vezes causando eventos zoonóticos (ZAKI et al. 2012). 
Os primeiros coronavírus identificados foram o HCoV-229E e o HCoV-OC43, em 1960, conhecidos como causadores do resfriado comum, podendo causar doenças respiratórias mais graves em lactentes e pacientes imunocomprometidos. Sendo ainda relatado por Peiris et al., (2003) como causa importante de pneumonia em recrutas militares.

Em 2003, o terceiro tipo de coronavírus descrito ficou conhecido como SARSCoV, sendo causador de uma pneumonia com risco de vida, a Síndrome Respiratória Aguda Grave (HOEK et al., 2004). Seu contágio se dá por gotículas e contato direto ou indireto (PEIRIS et al., 2003).

Em 2004, Hoek, et al. (2004) apresentou um estudo no qual fez uma descrição detalhada de um novo coronavírus humano, o HCoV-NL63, causador de doença respiratória aguda em crianças com menos de 1 ano de idade e em adultos imunocomprometidos.

Woo et al. (2005) descreveu em 2005 a sequência completa do genoma, características clínicas e epidemiologia molecular de outro novo tipo de vírus do grupo 2 de coronavírus humano, o coronavírus HKU1 (CoV-HKU1) (WOO et al., 2006).

Em 2012, foi isolado um vírus no escarro de um homem de 60 anos de idade que apresentou pneumonia aguda e subsequente insuficiência renal com resultado fatal na Arábia Saudita, representando uma nova espécie de betacoronavírus, o MERS-CoV, com quadro clínico semelhante ao do surto de síndrome respiratória aguda grave (SARS) em 2003 (ZAKI et al.. 2012). Sabe-se que esse vírus é comum em camelos dromedários, podendo ser essa a fonte de infecções humanas. Atualmente, existem medidas limitadas de quarentena e de biossegurança para reduzir a exposição dos seres humanos ao vírus (SABIR et al., 2015).

Em dezembro de 2019, uma série de casos de pneumonia de causa desconhecida, em pessoas expostas a frutos do mar ou mercado úmido, surgiu em Wuhan, na China, com apresentações clínicas muito semelhantes à pneumonia viral (HUANG et al., 2020). A partir desses casos, foram feitos estudos do sequenciamento genético do vírus causador, identificando-o como novo coronavírus 2019-nCoV (PERLMAN, 2020). Em 24 de janeiro de 2020, havia mais de 800 casos relatados, com uma taxa de mortalidade de 3\% (PERLMAN, 2020). 
Diante do exposto, podemos perceber que não é a primeira vez que o mundo vive um estado de pandemia devido à contaminação em massa por vírus, porém, com a globalização aumenta a chance de se espelhar cada vez por mais países, gerando mais medo e insegurança em toda a população que tem buscado cada vez mais informações e se proteger dessa nova ameaça.

\section{METODOLOGIA}

Trata-se de um estudo de revisão bibliográfica, temporal, com abordagem quantitativa de caráter retrospectivo, realizado por meio da consulta a bases de dados do site da Organização Mundial de Saúde (OMS), PubMed, Google Acadêmico e LILACS, usando as palavras-chave "coronavírus", "2019 novo coronavírus" ou "2019-nCoV".

\section{RESULTADOS E DISCUSSÕES}

Ao fazer uma retrospectiva histórica sobre o Coronavírus, observa-se que embora a maioria das infecções por coronavírus humano seja leve, as epidemias do coronavírus da síndrome respiratória aguda grave (SARS-CoV) e coronavírus da síndrome respiratória do Oriente Médio (MERS-CoV), causaram mais de 10.000 casos cumulativos nas últimas duas décadas, com taxas de mortalidade de $10 \%$ para SARS-CoV e 37\% para MERS-CoV (HUANG et al., 2020) e que o 2019-nCoV vem se assemelhando com estes dois tipos mais agressivos do vírus, gerando um estado de alerta assim como ocorreu com essas doenças nos seus períodos de surto global.

A SARS-Cov causou inicialmente em novembro de 2002, um surto de pneumonia atípica na China, afetando 792 pessoas e causando 31 mortes, sem resposta ao tratamento antimicrobiano empírico para pneumonia aguda ou típica 
adquirida na comunidade. A doença se espalhou pelo mundo, afetando pacientes na América do Norte, Europa e outros países asiáticos (PEIRIS et al., 2003).

Nesses pacientes diagnosticados com SARS-CoV, a queixa apresentada na maioria dos casos foi febre ou falta de ar. Tosse e mialgia estavam presentes em mais da metade deles, sintomas do trato respiratório superior, como rinorreia e dor de garganta em alguns, havendo relatos também de diarreia aquosa e anorexia em $10 \%$ dos pacientes. O tempo médio entre o início dos sintomas e a piora é de 8 dias, com as complicações da doença associadas com idade avançada, linfopenia grave, alanina aminotransferase prejudicada e atraso no início da ribavirina e esteróide. Com a mortalidade de cerca de $2 \%$ com o uso do regime de tratamento (PEIRIS et al., 2003). Porém, essa taxa pode passar de 50\% em pessoas idosas (MARRA, 2003). Resultando nesse período entre 2002 e 2004, num total de 800 mortes (ZAKI et al.. 2012). Na Figura abaixo, podemos observar a distribuição global de SARS.

Figura 1. Distribuição global de SARS.

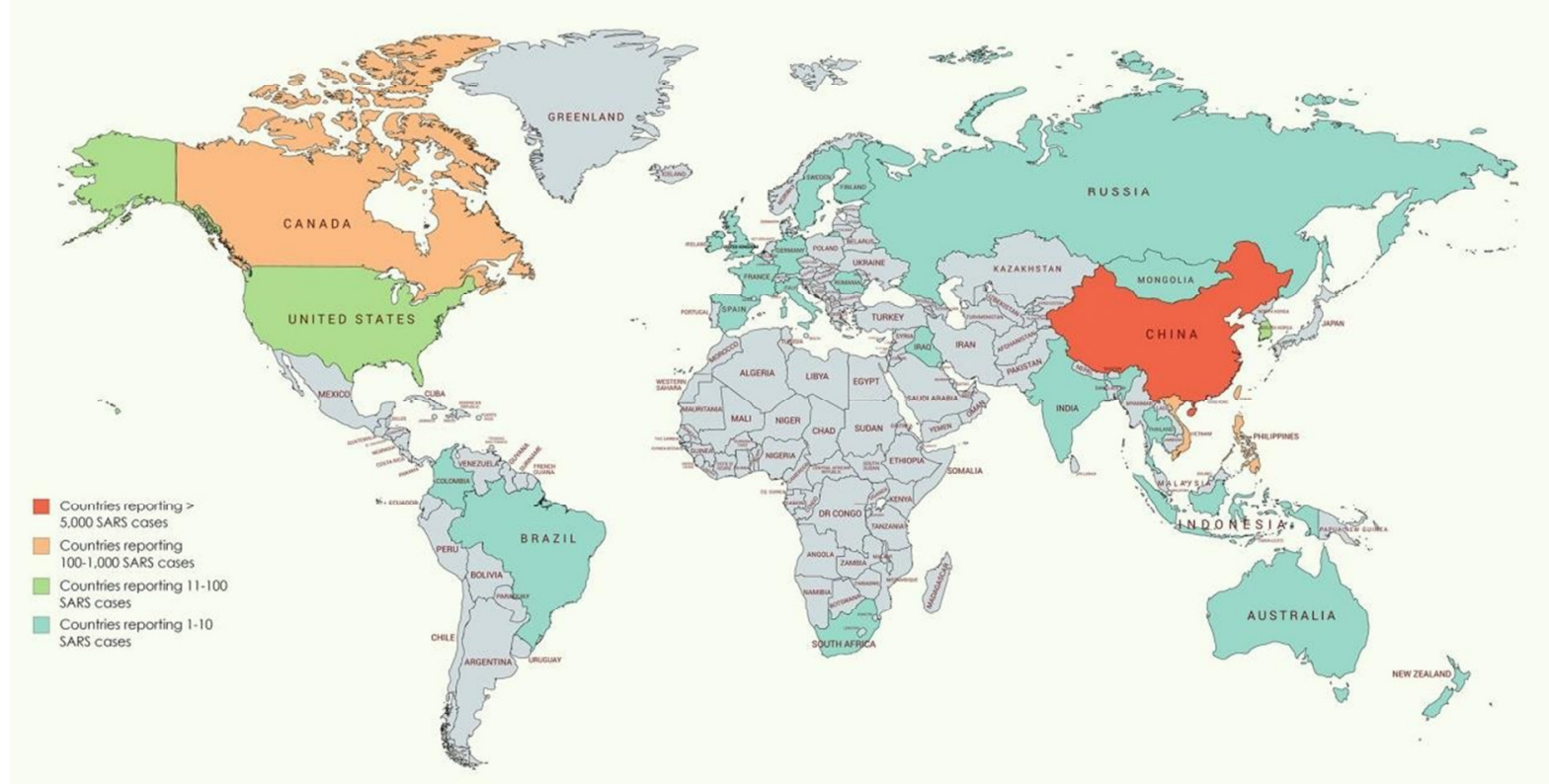

Fonte: HUI; ZUMLA (2019).

Em 2012, de acordo com a Organização Mundial Da Saúde (OMS) (2013), 50 casos foram confirmados em laboratório de infecção humana por coronavírus da síndrome respiratória do Oriente Médio (MERS-CoV) dos quais 30 foram a óbito. Já 
no ano de 2014, foram notificados a OMS (2014), a partir de abril, 572 casos de infecção por MERS-CoV, com confirmação em laboratório, dos quais 173 levaram os pacientes a óbito, atingindo países como a Jordânia, Líbano, Holanda, Emirados Árabes Unidos, Estados Unidos da América e mais 25 países.

De 1 de junho a 31 de julho de 2015, o MERS-CoV causou o maior surto fora da Península Arábica na República da Coréia, resultando em 186 casos confirmados de MERS e 38 mortes (AZHAR et al.. 2019).

Em 31 de julho de 2019, 2.458 casos de MERS confirmados em laboratório foram relatados à OMS, culminando com 848 óbitos (AZHAR et al.. 2019). Por isso, esses casos não devem ser negligenciados, mesmo que de acordo com Perlman (2020), o MERS-CoV não tenha sofrido uma mutação substancial para melhorar a infectividade humana desde que foi detectado em 2012, o que leva a pensar numa baixa virulência do vírus, os casos de MERS- CoV, pois novos casos continuam sendo relatados pela comunidade e hospitais da Península Arábica. Na figura 2 abaixo, pode-se observar a distribuição geográfica do MERS entre os anos de 2012 e 2018, como também a forma de transmissão do vírus no mundo. 
Fig. 2. Distribuição geográfica do MERS reportada à OMS (2012-2018).

\section{MERS-COV TRANSMISSION AND GEOGRAPHIC RANGE}
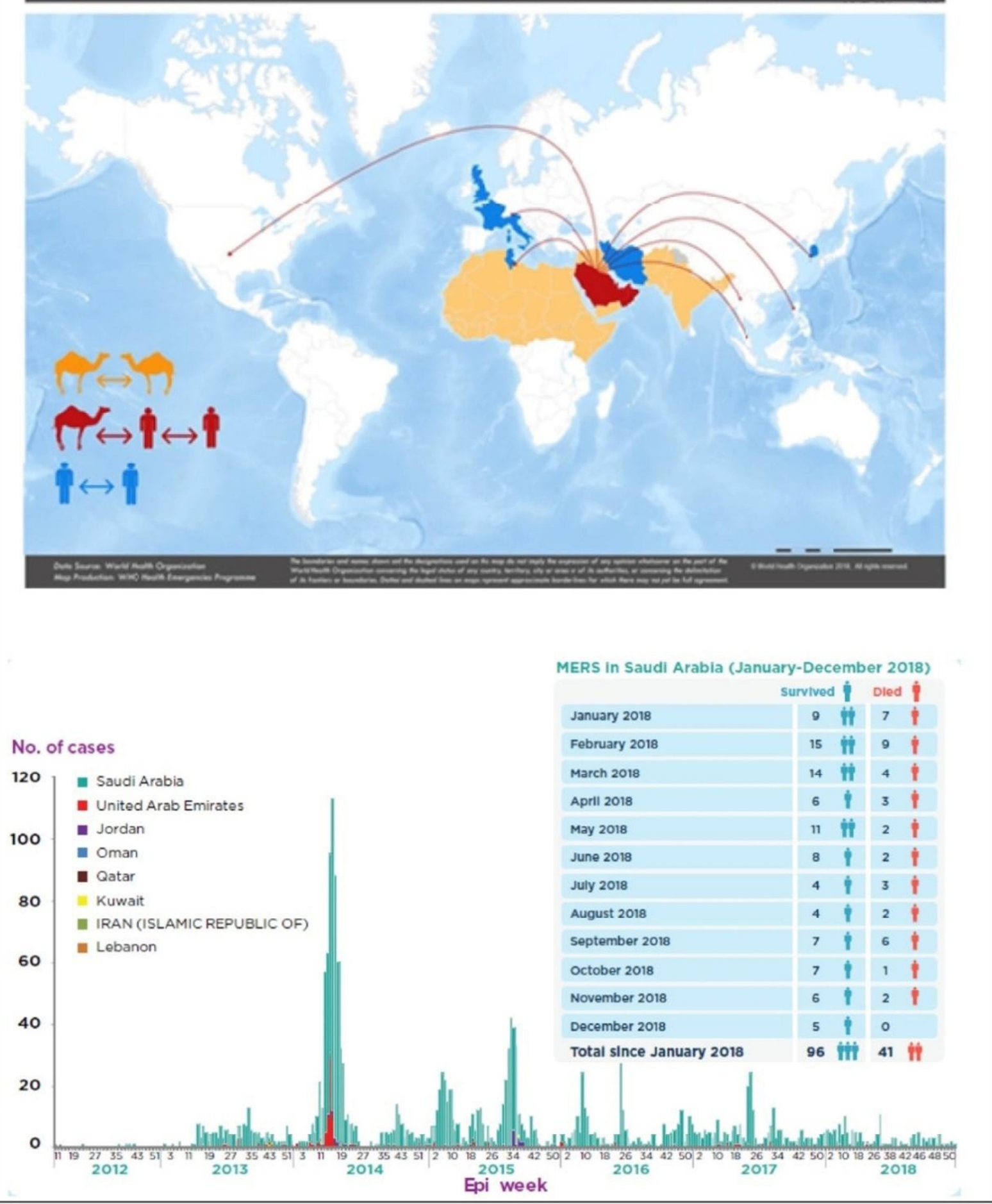

Fonte: AZHAR et al, 2019. 
Atualmente sabe-se de alguns dos fatores de risco para maior suscetibilidade à aquisição de infecções primárias por MERS-CoV, que são exposição direta ao dromedário na quinzena anterior ao início da doença, contato físico direto com camelos ou dromedário durante os 6 meses anteriores, diabetes mellitus e doenças cardíacas (AZHAR et al.. 2019).

No ano de 2019 teve início a distribuição dos casos do novo Coronavírus, o 2019 - nCoV, até o dia 2 de janeiro de 2020, 41 pacientes hospitalizados admitidos haviam sido identificados como tendo infecção por 2019-nCoV confirmada por laboratório. A maioria dos pacientes infectados era do sexo masculino (73\%) e menos da metade tinha doenças subjacentes $(32 \%)$, incluindo diabetes $(20 \%)$, hipertensão (15\%) e doenças cardiovasculares (15\%). A idade mediana dos pacientes foi de 49 anos. $66 \%$ deles foram expostos ao mercado de frutos do mar de Huanan. Um cluster de família foi encontrado (HUANG, C. et al., 2020). No entanto, os casos vêm aumentando numa velocidade muito alta, como podemos observar na figura 3 abaixo, na qual mostra cronologicamente como se deu a disseminação no mundo até 20 de janeiro de 2020 : 
Fig. 3. Cronologia dos estágios iniciais do surto de 2019-nCoV.

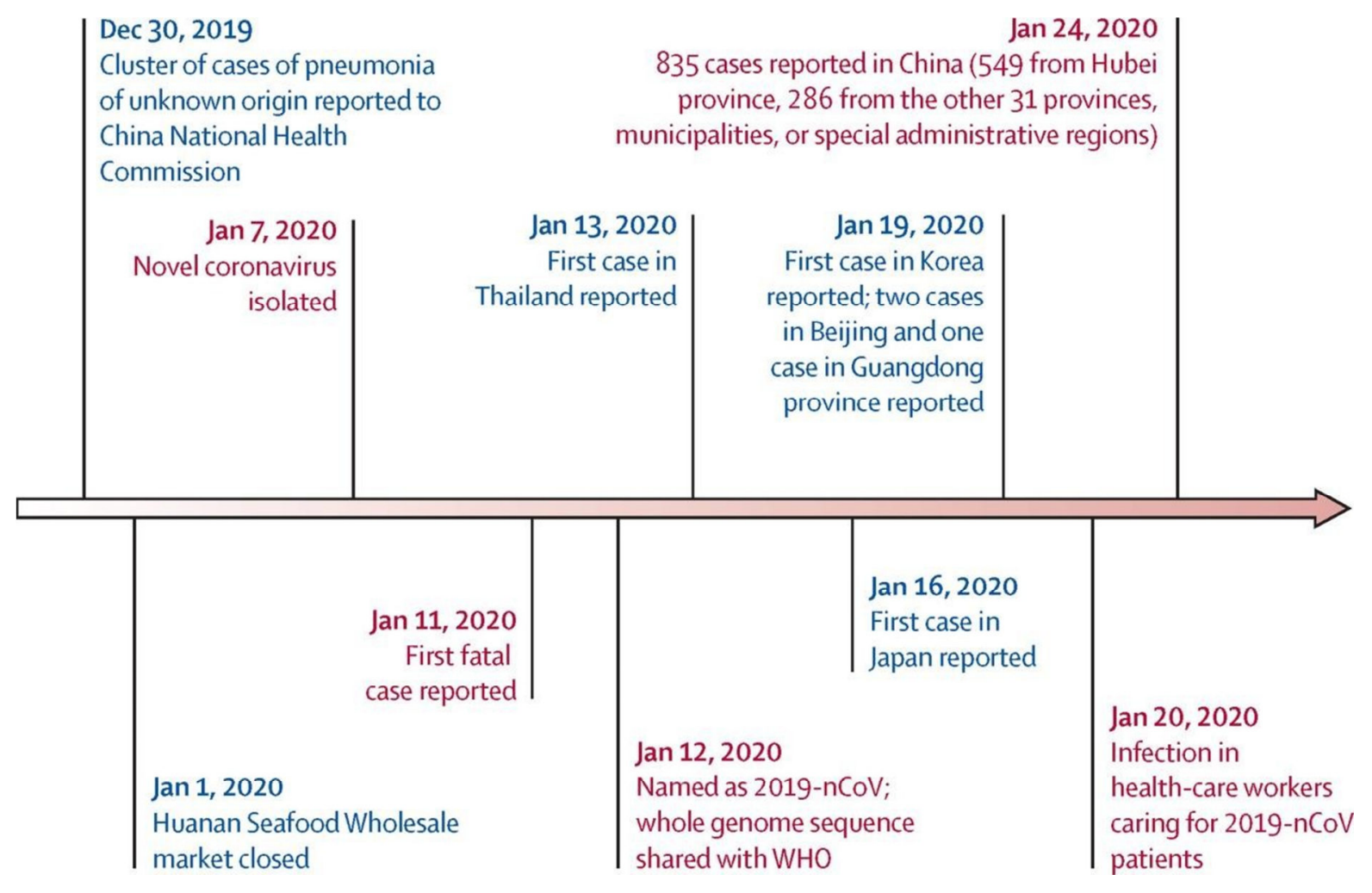

Fonte: WANG et al. 2020

Num documento enviado ao Journal of Hospital Infection, Beuy Joob e Viroj Wiwanitkit (2020) trazem que até 21 de fevereiro de 2020 haviam 35 pacientes infectados pelo 2019-nCoV na Tailândia, sendo um deles um profissional de saúde. A profissional em questão era uma enfermeira que teve contato com um paciente infectado com Coronavírus, mas que tinha sido diagnosticado com dengue, por isso nenhuma medida de controle de infecção respiratória estava em vigor para esse paciente. No entanto, o paciente hospitalizado com dengue recebeu posteriormente diagnóstico adicional de coronavírus. $\mathrm{O}$ documento ainda alerta para que seja feito controle de infecção respiratória para qualquer paciente com uma possível infecção, independentemente de apresentarem sintomas respiratórios evidentes e diz que o 2019-nCoV pode passar desapercebido em países tropicais por ocorrer em conjunto com uma doença infecciosa comum (como dengue) ou onde a infecção por COVID19 é diagnosticada como outra infecção mais comum (JOOB; WIWANITKIT, 2020). 
Em outro artigo, este com dados reportados de 22 de janeiro de 2020, enviado ao The New England Journal of Medicine, analisou os 425 primeiros casos confirmados de coronavírus em Wuhan, na província de Hubei na China. Entre eles, a idade média foi de 59 anos e $56 \%$ eram do sexo masculino. $55 \%$ dos casos com início antes de $1^{\circ}$ de janeiro de 2020 estava vinculada ao Mercado Atacadista de Frutos do Mar de Huanan, em comparação com $8,6 \%$ dos casos subsequentes. $O$ período médio de incubação foi de 5,2 dias. Nos estágios iniciais, a epidemia dobrava de tamanho a cada 7,4 dias. Com um intervalo serial médio de 7,5 dias, o número reprodutivo básico foi estimado em 2,2.

O primeiro caso 2019-nCoV na da América Latina foi no Brasil e teve diagnóstico molecular confirmado no dia 26 de fevereiro de 2020 pela equipe do Adolfo Lutz. O caso foi de um paciente infectado na Itália, possivelmente entre os dias 9 e 21 de fevereiro. Apenas dois dias após ter sido confirmado o caso na capital paulista, pesquisadores do Instituto Adolfo Lutz e das universidades de São Paulo (USP) e de Oxford (Reino Unido), conduzido por uma equipe coordenada por Claudio Tavares Sacchi, responsável pelo Laboratório Estratégico do Instituto Adolfo Lutz (LEIAL), e Jaqueline Goes de Jesus, pós-doutoranda na Faculdade de Medicina da USP e bolsista da Fapesp, publicaram a sequência completa do genoma viral, que recebeu o nome de SARS-CoV-2 (TOLEDO 2020).

Os dados foram divulgados no dia 28 de fevereiro no site Virological.org, um fórum de discussão e compartilhamento de dados entre virologistas, epidemiologistas e especialistas em saúde pública. Além de ajudar a entender como o vírus está se dispersando pelo mundo, esse tipo de informação é útil para o desenvolvimento de vacinas e testes diagnósticos. (TOLEDO 2020).

Anterior ao caso do Brasil, o genoma viral do 2019 - nCoV na China foi estudado, e mostrou que ele é 75 a $80 \%$ idêntico ao SARS-CoV e ainda mais semelhante a vários coronavírus de morcego. SARS-CoV e o MERS-CoV infectam células epiteliais intrapulmonares mais do que células das vias aéreas superiores, fazendo com que a transmissão ocorra principalmente em pacientes com doença reconhecida e não em pacientes com sinais leves e inespecíficos. Ao que tudo indica, o 2019-nCoV usa o mesmo receptor celular que SARS-CoV, esperando-se 
então que a transmissão ocorra apenas após o desenvolvimento de sinais de doença do trato respiratório inferior (PERLMAN, 2020).

Os sintomas comuns no início da doença foram febre, tosse e mialgia ou fadiga; os sintomas menos comuns foram produção de escarro, dor de cabeça, hemoptise e diarreia. (HUANG, C. et al., 2020). Em decorrência desses sintomas, muitos dos pacientes procuram departamentos de otorrinolaringologia (ORL), nos quais os procedimentos diagnósticos e terapêuticos envolvem contato direto com a mucosa do trato respiratório superior dos pacientes e/ou induzem os pacientes a tossir ou espirrar, gerando assim um risco particularmente alto para os profissionais de saúde e outros pacientes do setor de ORL, (LU et al..2020) pois, por mais que inicialmente se tenha descartado a transmissão de humano para humano, agora sabe-se que essa transmissão ocorre por meio de grandes gotas e contato direto ou indireto e menos por aerossóis e fomitos (PERLMAN, 2020).

Além desses sintomas iniciais, $55 \%$ dos 41 pacientes, que foram hospitalizado até 2 de janeiro de 2020, desenvolveram dispneia e o tempo médio desde o início da doença até o aparecimento da dispneia foi de 8 dia. 63\% desses pacientes apresentaram linfopenia nos exames laboratoriais. Todos apresentaram pneumonia com achados anormais na TC do tórax. As complicações incluíram síndrome do desconforto respiratório agudo (29\%), RNAemia (15\%), lesão cardíaca aguda (12\%) e infecção secundária (10\%). Dos 41 pacientes, 13 foram internados em UTI e 6 faleceram (14\%). Comparados com pacientes não internados em UTI, os pacientes em UTI apresentaram níveis plasmáticos mais elevados de IL2, IL7, IL10, GSCF, IP10, MCP1, MIP1A e TNFa (HUANG et al., 2020).

Em geral, o controle da propagação da infecção utiliza estratégias como aumento da conscientização e roupas de proteção, já que até então para o 2019nCoV o tratamento antiviral e a vacinação não estão disponíveis. Nos casos já confirmados, o objetivo é conter a disseminação adicional de infecções com medidas de hospitalização e quarentena, para aqueles que viajam ou viajaram recentemente da China para outros países (KHAN et al., 2020). 


\section{CONCLUSÃO}

Diante do exposto, os vírus do gênero Coronavírus, desde o início do século vêm levantando estados de alerta de saúde para a população mundial, e com o novo coronavírus, o 2019-nCoV, o mesmo vem ocorrendo. A nova pandemia vem gerando medo na população, devido ao impacto já vivido ao longo da história com as diversas pandemias, em especial com os outros Coronavírus.

$\mathrm{Na}$ infecção por SARS-Cov, em 2002, a mortalidade atingiu cerca de $2 \%$ com o uso do regime de tratamento, podendo passar de $50 \%$ em pessoas idosas e resultando entre 2002 e 2004, num total de 800 mortes.

$\mathrm{Na}$ infecção humana por coronavírus da síndrome respiratória do Oriente Médio (MERS-CoV), em 2012, a mortalidade foi de 60\%. Diminuindo para cerca de $30 \%$ em 2014, e em 2015, quando o MERS-CoV causou o maior surto fora da Península Arábica na República da Coréia, essa mortalidade foi de aproximadamente $20 \%$.

$\mathrm{Na}$ infecção com o novo Coronavírus, o 2019 - nCoV, até o dia 2 de janeiro de 2020, haviam 41 pacientes confirmados com a doença, de idade mediana de 49 anos, e a média de mortalidade foi $14 \%$. $66 \%$ deles foram expostos ao mercado de frutos do mar de Huanan. O período médio de incubação foi de 5,2 dias.

Até 21 de fevereiro de 2020 haviam 35 pacientes infectados pelo 2019-nCoV na Tailândia. E o primeiro caso 2019-nCoV na da América Latina foi no Brasil e teve diagnóstico molecular confirmado no dia 26 de fevereiro de 2020.

Essas infecções vem gerando também bastante insegurança, devido a sua rápida transmissibilidade, mesmo com todas as medidas de proteção que estão sendo adotadas, em decorrência da globalização. Nos estágios iniciais com o 2019 nCoV, a epidemia dobrava de tamanho a cada 7,4 dias.

Além disso, observou-se que a letalidade e gravidade da infecção é maior quando o paciente apresenta comorbidades, geralmente associadas a idade, sendo uma doença menos agressiva na maioria da população, porém continua sendo preocupante pois a infecção leva um grande número de indivíduos a hospitalização 
e devido seu alto grau de transmissibilidade. Porém, com a experiência de epidemias e pandemias passadas, com essa nova ameaça à saúde mundial, muitas medidas já foram tomadas, desde seu início, para minimizar seu impacto, mesmo que ainda desconhecido.

\section{REFERÊNCIAS BIBLIOGRÁFICAS}

AZHAR, E. I. et al. The Middle East Respiratory Syndrome (MERS). Infectious Disease Clinics Of North America, v. 33, n. 4, p.891-905, dez. 2019. Elsevier BV. http://dx.doi.org/10.1016/j.idc.2019.08.001.

HOEK, L D. et al. Identification of a new human coronavirus. Nature Medicine, v. 10, n. 4, p.368-373, 21 mar. 2004. Springer Science and Business Media LLC. http://dx.doi.org/10.1038/nm1024.

HUANG, C. et al. Clinical features of patients infected with 2019 novel coronavirus in Wuhan, China. The Lancet, v. 395, n. 10223, p.497-506, fev. 2020. Elsevier BV. http://dx.doi.org/10.1016/s0140-6736(20)30183-5.

HUI, D. S.c.; ZUMLA, Alimuddin. Severe Acute Respiratory Syndrome. Infectious Disease Clinics Of North America, v. 33, n. 4, p.869-889, dez. 2019. Elsevier BV. http://dx.doi.org/10.1016/j.idc.2019.07.001.

JOOB, Beuy; WIWANITKIT, Viroj. COVID-19 in medical personnel: observation from Thailand. Journal Of Hospital Infection. 27 fev. 2020. Disponível em: http://www.sciencedirect.com/science/article/pii/S0195670120300906. Acesso em: 01 mar. 2020.

KHAN, S. et al., Novel coronavirus, poor quarantine, and the risk of pandemic, Journal of Hospital Infection, https://doi.org/10.1016/j.jhin.2020.02.002.

$\mathrm{LI}, \mathrm{Q}$. et al. Early transmission dynamics in Wuhan, China, of novel coronavirus- infected pneumonia. New England Journal of Medicine, 2020.

LU, D. et al. Integrated infection control strategy tominimize nosocomial infection of corona virus disease 2019 among ENT healthcare workers, Journal ofHospital Infection, https://doi.org/10.1016/j.jhin.2020.02.018.

MARRA, M. A.. The Genome Sequence of the SARS-Associated Coronavirus. Science, v. 300, n. 5624, p.1399-1404, 1 maio 2003. American Association for the Advancement of Science (AAAS). http://dx.doi.org/10.1126/science.1085953.

Organização Mundial Da Saúde. Coronavírus da síndrome respiratória do Oriente Médio (MERS-CoV) - atualizado. 2014. http://www.who.int/csr/don/2014_05_15_mers/en/.

Organização Mundial da Saúde. Resumo do MERS-CoV e atualização da literatura - em 31 de maio de 2013. http://www.who.int/csr/disease/coronavirus_infections/update_20130531/en/.

PERLMAN, S. Another Decade, Another Coronavirus. New England Journal Of Medicine, [s.l.], v. 382, n. 8, p.760-762, 20 fev. 2020. Massachusetts Medical Society. 
http://dx.doi.org/10.1056/nejme2001126.

PEIRIS J. S. M. et al. Coronavírus como uma possível causa de síndrome respiratória aguda grave. Lancet 2003; 361: 1319 - 1325.

SABIR, J. S. et al.. Co-circulation of three camel coronavirus species and recombination of MERS-CoVs in Saudi Arabia. Science, v. 351, n. 6268, p.81-84, 17 dez. 2015. American Association for the Advancement of Science (AAAS). http://dx.doi.org/10.1126/science.aac8608.

SENHORAS, E. M.. CORONAVÍRUS E O PAPEL DAS PANDEMIAS NA HISTÓRIA HUMANA. Boletim de Conjuntura, Boa Vista, v. 1, n. 1, p.31-34, Jan, 2020. Disponível em: https://revista.ufrr.br/boca/article/view/Eloi/2799. Acesso em: 28 fev. 2020.

TOLEDO, K.. Tecnologia que sequenciou coronavírus em 48 horas permitirá monitorar epidemia em tempo real. Jornal da USP. São Paulo, 29 fev. 2020. Disponível em: https://jornal.usp.br/ciencias/ciencias-da-saude/tecnologia-que-sequenciou-coronavirus-em-48horas-permitira-monitorar-epidemia-em-tempo-real/. Acesso em: 01 mar. 2020.

WANG, C. et al. A novel coronavirus outbreak of global health concern. The Lancet, v. 395, n. 10223, p.470-473, fev. 2020. Elsevier BV. http://dx.doi.org/10.1016/s0140- 6736(20)30185-9.

WOO, P. C.Y. et al... Molecular diversity of coronaviruses in bats. Virology, v. 351, n. 1, p.180187, jul. 2006. Elsevier BV. http://dx.doi.org/10.1016/j.virol.2006.02.041.

ZAKI, A. M. et al..Isolation of a Novel Coronavirus from a Man with Pneumonia in Saudi Arabia. New England Journal Of Medicine, v. 367, n. 19, p.1814-1820, 8 nov. 2012. Massachusetts Medical Society. http://dx.doi.org/10.1056/nejmoa1211721. 